\title{
Mycobacterium orygis Lymphadenitis in New York, USA
}

\section{Luis A. Marcos, Eric D. Spitzer, Rahul Mahapatra, Yupo Ma, Tanya A. Halse, Joseph Shea, Michelle Isabelle, Pascal Lapierre, Vincent E. Escuyer}

Author affiliations: Stony Brook University, Stony Brook, New York, USA (L.A. Marcos, E.D. Spitzer, R. Mahapatra, Y. Ma); Wadsworth Center, New York State Department of Health, Albany, New York, USA (T.A. Halse, J. Shea, M. Isabelle, P. Lapierre, V.E. Escuyer)

DOI: https://doi.org/10.3201/eid2310.170490

We report a case of lymphadenitis caused by Mycobacterium orygis in an immunocompetent person in Stony Brook, New York, USA. Initial real-time PCR assay failed to provide a final subspecies identification within the $M$. tuberculosis complex, but whole-genome sequencing characterized the isolate as $M$. orygis.

G enomic analysis has previously shown that the $\mathrm{Myco}$ bacterium tuberculosis (MTB) complex comprises $>8$ distinct subgroups: $M$. tuberculosis, $M$. africanum, $M$. canettii, M. bovis, M. caprae, M. pinnipedii, M. microti, and M. mungi (1). M. orygis was first characterized in Africa and South Asia in 2012 based on examination of 22 isolates selected for the similarity of their IS6110 restriction fragment length polymorphism patterns to previously described oryx bacilli (2). Eleven of these isolates were from animals (a cow, a rhesus monkey, and types of antelope including oryx), and 11 were from humans (9 from South Asia). On the basis of single-nucleotide polymorphism (SNP) and region of difference (RD) analysis, van Ingen et al. concluded that these mycobacteria belonged to a phylogenetically distinct lineage of the clonal MTB complex (2). M. orygis is also distinguished by a mutation in gene Rv2042c (2) and a G1113A mutation in the gyrB gene (3).

We report a case of lymphadenitis caused by $M$. orygis in an immunocompetent person in Stony Brook, New York, USA. During July 2015, we diagnosed pneumonia in the upper lobe of the right lung in a woman, 71 years of age, who had a remote history of lymphoma. The condition was characterized by enlarged lymph nodes. The patient was born in Pakistan, moved to India at age 1, and emigrated to the United States $\approx 25$ years before onset; her preimmigration TB skin test was $<5 \mathrm{~mm}$ (bacillus Calmette-Guérin vaccinated), and chest radiograph results were negative. She drank unpasteurized milk while living in India.

We completed positron emission and computed tomography scans by using intravenous F-18 fluoro-2- deoxyglucose that detected hypermetabolic foci in the right axilla, subpectoral, subcarinal, and para hilar regions. QuantiFERON-TB Gold in-tube system (Quest Diagnostics, Inc., Lyndhurst, NJ, USA) test result was positive (TB antigen minus nil value $3.58 \mathrm{IU} / \mathrm{Ml}$, mitogen minus nil value 8.5 $\mathrm{IU} / \mathrm{mL}$ ). Three induced sputum samples for acid-fast bacilli smear and cultures were negative. Because of the patient's history of lymphoma, we biopsied the subpectoral lymph node. Histopathology revealed diffuse large caseating granulomas with extensive central necrosis, small lymphocytes, plasma cells, and histiocytes. Grocott's Methenamine Silver Stain (Ventana Medical Systems, Inc. Tucson, AZ, USA), and acid-fast bacilli stains did not detect organisms. Bacterial and fungal cultures were negative.

Mycobacterium Growth Indicator Tube (MGIT) system turned positive on day 29 , and the isolate was identified as MTB complex by probe hybridization (Hologic, Inc. San Diego, CA, USA). Further testing at the New York State Department of Health with real-time PCR using primers/ probes specific for 4 MTB regions of difference yielded an inconclusive pattern (4). Results were positive for RD1 and RD4, but negative for RD9 and RD12; subspecies identification was initially reported as "inconclusive" because this pattern did not match the signature patterns used to determine MTB complex species with this assay.

We performed a whole-genome sequencing assay that confirmed the absence of RD9 and RD12 and identified the isolate as $M$. orygis, as reported by Shea et al (5). This isolate belonged to Spogliotype International Type 587, contained the specific gyrB SNP at position 1113 (3), and lacked resistance-associated mutations, suggesting susceptibility to all tested current antituberculosis agents. The patient received first-line, 4-drug therapy.

$M$. orygis infections in humans have been rarely reported. In Australia, of 1,763 case-patients diagnosed with MTB complex infection, 8 causative pathogens were identified as M. orygis; all of the patients were born in India (6). In New Zealand, Dawson et al. used advanced molecular techniques to demonstrate a transmission of $M$. orygis from a human, who emigrated from India, to a cow (7).

$M$. orygis infection may be underreported in the literature because cases may be identified as MTB complex or misidentified as M. africanum or M. bovis (8). Through whole-genome sequencing, the New York State Department of Health identified 8 additional cases of $M$. orygis of 6,322 MTB complex isolates from New York tested (3 pulmonary, 2 lymph node, and 2 abscess samples) that were received during 2005-2016 but were initially misidentified (data not shown). All patients were from India, Pakistan, or Nepal and had moved to the United States. SNP analysis indicated that the M. orygis isolates were genetically similar, but all were distant from other members of the MTB complex (Figure) and contained the G1113A mutation in $g y r B$. 
Figure. Maximumlikelihood single-nucleotide polymorphism (SNP) tree of 8 Mycobacterium orygis and $1 \mathrm{M}$. caprae isolates obtained from patients in New York, USA. Alignment of 5,242 total SNP positions was calculated by using PhyML version 20111216 (http://www.atgc-montpellier.fr/ phyml/) general time reversible plus gamma model under 8 categories with best of nearest-neighbor interchange, subtree pruning, and regrafting with 5 random starting trees. Included in the tree are $M$. tuberculosis H37Rv (GenBank accession no. NC_000962), $M$. orygis (accession no. APKD01000001.1), M. bovis (accession no. NC_002945.3), $M$. africanum (accession no.

NC_015758.1), and M. microti (ATCC 35782) reference sequences. SNP analysis of the isolate from the patient described in this study (LN 2016) with 7 other M. orygis strains identified at the New York State Department of Health showed differences ranging from 170 (closest) to 323 (farthest) SNPs. By comparison, the closest non-M. orygis M. tuberculosis complex species is M. microti (1,880 SNPs). All M. orygis strains are grouped with $100 \%$ bootstrap support. Scale bar indicates average number of substitutions per site. Specimen sources: Resp, respiratory; Absc, abcess; LN, lymph node (BioProject ID PRJNA389109 containing BioSample accessions SAMN07190143-50).

The number of SNPs separating the 8 M. orygis isolates was 106-323, which excludes their belonging to an epidemiologic transmission cluster (9) and strongly suggests that the infections were independently acquired.

We found no previous reports of $M$. orygis originating in the Americas; the most notable epidemiologic risk factor in this patient was prior residency in India, where M. orygis was found in a variety of animals (10). Because all organisms in the MTB complex have a distinct host preference, it is possible that $M$. orygis is mostly present in animals and few cases occur in humans, similar to $M$. bovis. This case demonstrates the value of molecular methodologies such as whole-genome sequencing for providing more detailed insight into the clinical and epidemiologic aspects of the MTB complex.

Dr. Marcos is an associate professor of clinical medicine at Stony Brook University in New York, USA. His research interests include epidemiology of emerging infectious diseases, tickborne diseases, and global health.

\section{References}

1. Brosch R, Gordon SV, Marmiesse M, Brodin P, Buchrieser C, Eiglmeier K, et al. A new evolutionary scenario for the Mycobacterium tuberculosis complex. Proc Natl Acad Sci U S A. 2002;99:3684-9. http://dx.doi.org/10.1073/pnas.052548299

2. van Ingen J, Rahim Z, Mulder A, Boeree MJ, Simeone R, Brosch R, et al. Characterization of Mycobacterium orygis as M. tuberculosis complex subspecies. Emerg Infect Dis. 2012; 18:653-5. http://dx.doi.org/10.3201/eid1804.110888
3. Huard RC, Fabre M, de Haas P, Lazzarini LCO, van Soolingen D, Cousins D, et al. Novel genetic polymorphisms that further delineate the phylogeny of the Mycobacterium tuberculosis complex. J Bacteriol. 2006;188:4271-87. http://dx.doi.org/10.1128/JB.01783-05

4. Halse TA, Escuyer VE, Musser KA. Evaluation of a single-tube multiplex real-time PCR for differentiation of members of the Mycobacterium tuberculosis complex in clinical specimens. J Clin Microbiol. 2011;49:2562-7. http://dx.doi.org/10.1128/ JCM.00467-11

5. Shea J, Halse TA, Lapierre P, Shudt M, Kohlerschmidt D, Van Roey P, et al. Comprehensive whole-genome sequencing and reporting of drug resistance profiles on clinical cases of Mycobacterium tuberculosis in New York State. J Clin Microbiol. 2017;55:1871-82. http://dx.doi.org/10.1128/JCM.00298-17

6. Lavender CJ, Globan M, Kelly H, Brown LK, Sievers A, Fyfe JA, et al. Epidemiology and control of tuberculosis in Victoria, a lowburden state in south-eastern Australia, 2005-2010. Int J Tuberc Lung Dis. 2013;17:752-8. http://dx.doi.org/10.5588/ijtld.12.0791

7. Dawson KL, Bell A, Kawakami RP, Coley K, Yates G, Collins DM. Transmission of Mycobacterium orygis (M. tuberculosis complex species) from a tuberculosis patient to a dairy cow in New Zealand. J Clin Microbiol. 2012;50:3136-8. http://dx.doi.org/10.1128/ JCM.01652-12

8. Tortoli E. Microbiological features and clinical relevance of new species of the genus Mycobacterium. Clin Microbiol Rev. 2014;27:727-52. http://dx.doi.org/10.1128/CMR.00035-14

9. Walker TM, Ip CL, Harrell RH, Evans JT, Kapatai G, Dedicoat MJ, et al. Whole-genome sequencing to delineate Mycobacterium tuberculosis outbreaks: a retrospective observational study. Lancet Infect Dis. 2013;13:137-46. http://dx.doi.org/10.1016/S14733099(12)70277-3

10. Thapa J, Paudel S, Sadaula A, Shah Y, Maharjan B, Kaufman GE, et al. Mycobacterium orygis-associated tuberculosis in free-ranging rhinoceros, Nepal, 2015. Emerg Infect Dis. 2016;22:570-2. http://dx.doi.org/10.3201/eid2203.151929 
Address for correspondence: Luis A. Marcos, Stony Brook University Health Sciences Center School of Medicine, Internal Medicine, Division of Infectious Disease, 101 Nicolls Rd, HSC T 16, Rm 027J, Stony Brook, NY 11794, USA; email: luis.marcos@stonybrookmedicine.edu

\section{Ross River Virus Seroprevalence, French Polynesia, 2014-2015}

\section{Maite Aubry, Anita Teissier, Michael Huart, Sébastien Merceron, Jessica Vanhomwegen, Claudine Roche, Anne-Laure Vial, Sylvianne Teururai, Sébastien Sicard, Sylvie Paulous, Philippe Desprès, Jean-Claude Manuguerra, Henri-Pierre Mallet, Didier Musso, Xavier Deparis, Van-Mai Cao-Lormeau}

\begin{abstract}
Author affiliations: Institut Louis Malardé, Tahiti, French Polynesia (M. Aubry, A. Teissier, C. Roche, S. Teururai, D. Musso, V.-M. Cao-Lormeau); Centre D'épidémiologie et de Santé Publique des Armées, Marseille, France; and Unité Mixte de Recherche Sciences Economiques et Sociales de la Santé et Traitement de I'Information Médicale, Marseille (M. Huart, S. Sicard, X. Deparis); Institut de la Statistique de la Polynésie Française, Tahiti; and Institut National de la Statistique et des Études Économiques, Sainte Clotilde, Réunion (S. Merceron); Institut Pasteur, Paris, France (J. Vanhomwegen, S. Paulous, J.-C. Manuguerra); Direction Départementale de la Cohésion Sociale et de la Protection des Populations, Yonne, France (A.-L. Vial); Direction de la Santé de la Polynésie Française, Tahiti (A.-L. Vial, H.-P. Mallet); Université de La Réunion, Sainte Clotilde, France; and Unité Mixte de Recherche Processus Infectieux en Milieu Insulaire Tropical, Sainte Clotilde (P. Desprès)
\end{abstract}

DOI: https://doi.org/10.3201/eid2310.170583

Ross River virus (RRV), spread by Aedes and Culex mosquitoes, is the most commonly transmitted arbovirus in Australia. A serosurvey of blood donors in French Polynesia during 2011-2013 suggested that RRV circulated without being detected. We report RRV circulation in French Polynesia based on further screening of blood samples collected during 2014-2015.

$\mathrm{R}$ oss River virus (RRV), an alphavirus of the family Togaviridae, is an arbovirus transmitted by Aedes and
Culex mosquito species (1). Symptoms of RRV infections mainly consist of fever, arthralgia, and rash. RRV was first isolated in North Queensland in 1959 and has become the most common arboviral disease in Australia (2). RRV outbreaks were reported during 1979-1980 in Pacific Island countries and territories (PICTs) including Fiji, Cook Islands, American Samoa, and New Caledonia (1).

In French Polynesia (FP), a territory of the Pacific region with $\approx 270,000$ inhabitants, dengue virus (DENV) was the only arbovirus detected until identification of the Zika virus (ZIKV), then chikungunya virus (CHIKV), causing outbreaks during 2013-2014 and 2014-2015, respectively (3). Although RRV infections have never been reported in FP, a serosurvey of blood donors during 20112013 suggested that RRV has circulated without being detected (4). In this study, we report additional evidence of RRV circulation in FP based on further screening of blood samples collected during 2014-2015 and previously used for a ZIKV serosurvey (5).

We tested 3 groups of participants randomly sampled in FP for the presence of anti-RRV IgG by using recombinant antigen-based indirect ELISA (patent no.WO2012076715A1) and microsphere multiplex immunoassay (patent no. WO2013083847A2) (4,5). The first group consisted of 196 participants recruited during February-March 2014 in 8 of the most inhabited islands of the 5 FP archipelagos (archipelagos listed in parentheses): Tahiti and Moorea (Society), Rangiroa and Makemo (Tuamotu), Nuku Hiva and Hiva Oa (Marquesas), Rurutu (Austral), and Rikitea (Gambier). The second group included 700 participants recruited during September-November 2015 on the 2 most inhabited islands of FP: Tahiti and Moorea. The third group consisted of 476 schoolchildren recruited during May-June 2014 on Tahiti. The Ethics Committee of French Polynesia approved recruitment of participants and processing of blood samples (approval no. 60/CEPF-06/27/2013). We analyzed seroprevalence data by using the Fisher exact test, and considered $\mathrm{p}$ values $<0.05$ as statistically significant.

RRV seropositivity rates among participants sampled in the 5 archipelagos in 2014 ranged from 16\% to $49 \%$ (average $35 \%$ ), and were significantly different between the Society and Marquesas $(\mathrm{p}=0.036)$, Tuamotu and Marquesas $(\mathrm{p}=0.001)$, and Tuamotu and Austral-Gambier $(\mathrm{p}=0.002)$ Islands (Table). In the Society Islands, screening of additional participants in 2015 did not lead to a significant difference in RRV seroprevalence (18\%) compared with the 1 observed in the participants recruited in $2014(27 \%)(p=0.125)$. In contrast, RRV seroprevalence among schoolchildren (1\%) was significantly lower than in the general population from the Society Islands, during both 2014 and 2015 ( $\mathrm{p}<0.0001)$. We compared 2 groups in the general population in 2014 and 2015. Rates of participants whose samples were RRVseropositive and who were born or arrived in FP before 